\title{
SIMPLE MASS FORMULAS ON SHIMURA VARIETIES OF PEL-TYPE
}

\author{
CHIA-FU YU
}

\begin{abstract}
We give a unified formulation of a mass for arbitrary abelian varieties with PEL-structures and show that it equals a weighted class number of a reductive $\mathbb{Q}$-group $G$ relative to an open compact subgroup $U$ of $G\left(\mathbb{A}_{f}\right)$, or simply called an arithmetic mass. We classify the special objects for which our formulation remains valid over algebraically closed fields. As a result, we show that the set of basic points in a mod $p$ moduli space of PEL-type with a local condition (and a mild condition subject to the Hasse principle) can be expressed as a double coset space and its mass equals an arithmetic mass. The moduli space does not need to have good reduction at $p$. This generalizes a well-known result for superspecial abelian varieties.
\end{abstract}

\section{INTRODUCTION}

The idea of using elliptic curves to study class numbers might go back to Kronecker or even to Gauss. The celebrated Eichler-Deuring mass formula says

$$
\sum_{E \in \Lambda_{p}} \frac{1}{\# \operatorname{Aut}(E)}=\frac{p-1}{24}
$$

where $\Lambda_{p}$ is the set of isomorphism classes of supersingular elliptic curves over $\overline{\mathbb{F}}_{p}$. If we let $\mathbf{B}:=B_{p, \infty}$ be the definite quaternion algebra over $\mathbb{Q}$ of discriminant $p$ and $O_{\mathbf{B}}$ a maximal order, then $\# \Lambda_{p}$ equals the class number of $\mathbf{B}$. Using the adelic language, let $G^{\prime}$ be the group scheme over $\mathbb{Z}$ attached to the multiplicative group $O_{\mathbf{B}}^{\times}$; then one has the following natural bijection:

$$
\Lambda_{p} \simeq G^{\prime}(\mathbb{Q}) \backslash G^{\prime}\left(\mathbb{A}_{f}\right) / G^{\prime}(\hat{\mathbb{Z}}),
$$

where $\hat{\mathbb{Z}}=\prod_{p} \mathbb{Z}_{p}$ and $\mathbb{A}_{f}=\hat{\mathbb{Z}} \otimes_{\mathbb{Z}} \mathbb{Q}$ is the finite adèle ring of $\mathbb{Q}$.

We write $\operatorname{mass}\left(\Lambda_{p}\right)$ for the left hand side of (1.1), and call it the (geometric) mass of $\Lambda_{p}$. One shows that mass $\left(\Lambda_{p}\right)$ equals an arithmetically defined mass for the group $G^{\prime}$ relative to the open compact subgroup $G^{\prime}(\hat{\mathbb{Z}})$. The definition is as follows. For an $\mathbb{R}$-anisotropic reductive $\mathbb{Q}$-group $G$, and an open compact subgroup $U$ of $G\left(\mathbb{A}_{f}\right)$, the (arithmetic) mass for $G$ relative to $U$ is defined to be

$$
\operatorname{mass}(G, U):=\sum_{c} \frac{1}{\# \Gamma_{c}}
$$

where $c$ runs through a complete set of representatives for the double coset space $G(\mathbb{Q}) \backslash G\left(\mathbb{A}_{f}\right) / U$, and $\Gamma_{c}:=G(\mathbb{Q}) \cap c U c^{-1}$.

The analogous result of the Eichler-Deuring mass formula for the Siegel moduli spaces is generalized in Ekedahl [3]. Let $\Lambda_{g, p}$ be the set of the isomorphism classes

Date: June 22, 2007. The research is partially supported by 95-2115-M-001-004. 
of $g$-dimensional superspecial principally polarized abelian varieties over $\overline{\mathbb{F}}_{p}$. An abelian variety over an algebraically closed field $k \supset \mathbb{F}_{p}$ is called supersingular if it is isogenous to a product of supersingular elliptic curves. It is called superspecial if it is isomorphic to a product of supersingular elliptic curves. It is well-known that $\Lambda_{g, p}$ is a finite set. Let $G_{g}^{\prime}$ be the group scheme over $\mathbb{Z}$ obtained from $M_{g}\left(O_{\mathbf{B}}\right)$ with equations given by $g^{*} g=1$, where $g \mapsto g^{*}$ is the standard involution. Similar to the bijection (1.2), one has (see [3, also see [7, Theorem 2.10, p. 144])

$$
\Lambda_{g, p} \simeq G_{g}^{\prime}(\mathbb{Q}) \backslash G_{g}^{\prime}\left(\mathbb{A}_{f}\right) / G_{g}^{\prime}(\hat{\mathbb{Z}}) \quad \text { and } \operatorname{mass}\left(\Lambda_{g, p}\right)=\operatorname{mass}\left(G_{g}^{\prime}, G_{g}^{\prime}(\hat{\mathbb{Z}})\right),
$$

where

$$
\operatorname{mass}\left(\Lambda_{g, p}\right):=\sum_{(A, \lambda) \in \Lambda_{g, p}} \frac{1}{\# \operatorname{Aut}(A, \lambda)} .
$$

Applying a formula for $\operatorname{mass}\left(G_{g}^{\prime}, G_{g}^{\prime}(\hat{\mathbb{Z}})\right)$ in Hashimoto-Ibukiyama [6, Proposition 9, p. 568] to the second formula in (1.4), Ekedahl obtained the geometric mass formula

$$
\sum_{(A, \lambda) \in \Lambda_{g, p}} \frac{1}{\# \operatorname{Aut}(A, \lambda)}=\frac{(-1)^{g(g+1) / 2}}{2^{g}}\left\{\prod_{k=1}^{g} \zeta(1-2 k)\right\} \cdot \prod_{k=1}^{g}\left\{\left(p^{k}+(-1)^{k}\right\} .\right.
$$

To generalize geometric mass formulas to Shimura varieties of PEL-type, the first step is to look for a set $\Lambda$ of certain special points, such as $\Lambda_{g, p}$ above, in the reduction $\mathcal{M} \otimes \overline{\mathbb{F}}_{p}$ modulo $p$ of a moduli space $\mathcal{M}$ of PEL-type so that $\Lambda$ can be parameterized by a double coset space of a reductive group, such as $G_{g}^{\prime}$ above. Naturally one would consider good reduction cases (as little is known in bad reduction cases) and consider the set of basic abelian varieties which are "minimal" in the sense of Oort 13. Basic abelian varieties, defined in Kottwitz [8, are those which land in a minimal Newton polygon stratum of the moduli space $\mathcal{M} \otimes \overline{\mathbb{F}}_{p}$. The minimal basic points in $\mathcal{M} \otimes \overline{\mathbb{F}}_{p}$ play the same role as superspecial points in the Siegel modular varieties; particularly they form a finite set. Note that the basic locus has positive dimension in general; thus there is no natural definition for the mass of the basic locus.

It turns out that the correspondence in (1.4) can be more flexible, even in the Siegel modular varieties. We do not need to assume that polarizations are principal nor that supersingular abelian varieties are "minimal", that is, superspecial. All we need to do is imposing a type of isomorphism class on the associated polarized $p$-divisible groups. This is proved in [16] for the Hilbert-Blumenthal varieties and in [17 for the Hilbert-Siegel modular varieties. It is conceivable that the same correspondence should hold for basic points in a PEL-type Shimura variety.

In this paper we give a unified formulation of the (geometric) mass mass $(\Lambda)$ for arbitrary abelian varieties with additional structures over arbitrary (finitely generated) fields. We also show that it equals the arithmetic mass defined by a pair $(G, U)$ which can be explicitly described; see Section 2 for precise statements. The description, though being surprisingly simple, is by no means obvious. It relies on the deep results of Zarhin, Faltings and de Jong on the endomorphisms of abelian varieties, Tate modules, and $p$-divisible groups; see [18, 4] (cf. [5]) and 2, Theorem 2.6]. We call the formula established in Theorem 2.2 the simple mass formula. The simple mass formula connects masses between the geometric side and the arithmetic 
side; but it provides no clue of computing either side explicitly. It serves as a comparison theorem between masses arising from different natures in a very general setting. For example, one can use it to prove a geometric mass formula starting from a known arithmetic mass formula and vice versa, or to verify an arithmetic mass formula by geometry and vice versa. The geometric mass formula (1.5) is an example. Another example is given in [17] where the mass for superspecial abelian varieties with real multiplication is calculated. It is worth to note that a geometric mass then shares good properties as an arithmetic mass does. For example, it has a simple relation between different levels and it can be calculated through local volume computation.

In some sense the simple mass formula says arithmetic properties of abelian varieties. For generic abelian varieties, the simple mass formula provides no information; thus those are not interesting cases. In the extreme cases such as CM abelian varieties or supersingular abelian varieties, the simple mass formula provides non-trivial information and becomes interesting. In Section 3 we study a class of special abelian varieties in question which we call of arithmetic type. Those include CM abelian varieties and supersingular abelian varieties. Strictly speaking, this notion is for an abelian variety $\underline{A}=(A, \lambda, \iota)$ with additional structures, not for the abelian variety $A$ itself. Furthermore, it is a "geometric" property that is independent of ground fields over which the object is defined. The (equivalent) definitions are given in Def. 3.1 and Def. 3.8, For these abelian varieties the hidden Galois structure required in the mass formula is superfluous; thus the description in Theorem 2.2 can be extended in a geometric setting. Namely, the ground field can be an algebraically closed field; see Theorem 3.12 . This explains why a good formulation of the mass for supersingular abelian varieties in characteristic $p$ (more generally, basic abelian varieties with additional structures) or CM abelian varieties in characteristic zero is possible. Some detail discussion is included for motivating the definitions and clarifying the notion of abelian varieties of arithmetic type. We remark that the description for CM abelian varieties in characteristic zero is wellknown. This has been playing an important role on explicit reciprocity laws in class field theory, known as the main theorem of complex multiplication. It is interesting to describe the Galois action on the class space in terms of Hecke translation in our setting. This might lead an interesting explicit reciprocity law.

In the last section we classify abelian varieties $\underline{A}$ with additional structures which are of arithmetic type. In the case of characteristic zero, the possibility occurs only when every simple factor of the semi-simple algebra $(B, *)$ is of second kind. Under this condition, every object $\underline{A}$ of arithmetic type is essentially a self product of a simple CM abelian variety (Proposition 4.2). In the case of characteristic $p$, we show that an object $\underline{A}$ is of arithmetic type if and only if it is basic in the sense of Kottwitz 8] (Proposition 4.5). Applying the results in Sections 2 and 3 , we complete the first step of a geometric mass formula for basic points in Shimura varieties of PEL-type; see Theorem 4.6.

Acknowledgments. The author thanks J.-K. Yu for helpful discussions in an early stage of this work. Main part of the manuscript is prepared during the author's stay at MPIM in Bonn in the fall of 2005. He acknowledges the Institute for kind 
hospitality and excellent working environment.

\section{Simple MASS FORMULAS}

2.1. Let $B$ be a finite-dimensional semi-simple algebra over $\mathbb{Q}$ with a positive involution $*$, and $O_{B}$ be any order of $B$ stable under $*$.

A polarized abelian $O_{B}$-variety is a triple $\underline{A}=(A, \lambda, \iota)$, where $(A, \lambda)$ is a polarized abelian variety and $\iota: O_{B} \rightarrow \operatorname{End}(A)$ is a ring monomorphism such that $\lambda \iota\left(a^{*}\right)=$ $\iota(a)^{t} \lambda$ for all $a \in O_{B}$. For any $\underline{A}$ and any prime $\ell$ (not necessarily invertible in the ground field), we write $\underline{A}(\ell)$ for the associated $\ell$-divisible group with additional structures $\left(A\left[\ell^{\infty}\right], \lambda_{\ell}, \iota_{\ell}\right)$, where $\lambda_{\ell}$ is the induced quasi-polarization from $A\left[\ell^{\infty}\right]$ to $A^{t}\left[\ell^{\infty}\right]=A\left[\ell^{\infty}\right]^{t}$ (the Serre dual), and $\iota_{\ell}: O_{B} \otimes \mathbb{Z}_{\ell} \rightarrow \operatorname{End}\left(A\left[\ell^{\infty}\right]\right)$ the induced ring monomorphism.

For any two $\underline{A}_{1}$ and $\underline{A}_{2}$ over a field $k$, denote by

- Q-isom $k\left(\underline{A}_{1}, \underline{A}_{2}\right)\left(\operatorname{resp} . \operatorname{Isom}_{k}\left(\underline{A}_{1}, \underline{A}_{2}\right)\right)$ the set of $O_{B}$-linear quasi-isogenies (resp. isomorphisms) $\varphi: A_{1} \rightarrow A_{2}$ over $k$ such that $\varphi^{*} \lambda_{2}=\lambda_{1}$, and

- $\mathrm{Q}$-isom $k\left(\underline{A}_{1}(\ell), \underline{A}_{2}(\ell)\right)$ (resp. $\left.\operatorname{Isom}_{k}\left(\underline{A}_{1}(\ell), \underline{A}_{2}(\ell)\right)\right)$ the set of $O_{B} \otimes \mathbb{Z}_{\ell^{-}}$ linear quasi-isogenies (resp. isomorphisms) $\varphi: A_{1}\left[\ell^{\infty}\right] \rightarrow A_{2}\left[\ell^{\infty}\right]$ such that $\varphi^{*} \lambda_{2}=\lambda_{1}$.

Let $k$ be a field of finite type over its prime field, and let $x:=\underline{A}_{0}=\left(A_{0}, \lambda_{0}, \iota_{0}\right)$ be a fixed polarized abelian $O_{B}$-variety over $k$. Denote by $\Lambda_{x}(k)$ the set of isomorphisms classes of polarized abelian $O_{B}$-varieties $\underline{A}$ over $k$ such that

(i) $\left(I_{\ell}\right): \operatorname{Isom}_{k}\left(\underline{A}_{0}(\ell), \underline{A}(\ell)\right) \neq \emptyset$ for all $\ell$, and

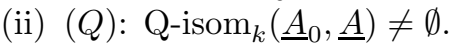

Let $G_{x}$ be the automorphism group scheme over $\mathbb{Z}$ associated to $\underline{A}_{0}$; for any commutative ring $R$, its group of $R$-valued points is

$$
G_{x}(R)=\left\{g \in \operatorname{End}_{O_{B}}\left(A_{0 / k}\right) \otimes R \mid g^{\prime} g=1\right\},
$$

where $g \mapsto g^{\prime}$ is the Rosati involution induced by $\lambda_{0}$. By definition,

$$
G_{x}(\mathbb{Q})=\mathrm{Q}^{-\operatorname{isom}_{k}}\left(\underline{A}_{0}, \underline{A}_{0}\right) .
$$

For the group of $\mathbb{Q}_{\ell}$-valued points of $G_{x}$, we have

Theorem 2.1 (Zarhin, Faltings, de Jong). Notations as above, one has the natural isomorphisms

$$
G_{x}\left(\mathbb{Z}_{\ell}\right)=\operatorname{Isom}_{k}\left(\underline{A}_{0}(\ell), \underline{A}_{0}(\ell)\right) \quad \text { and } \quad G_{x}\left(\mathbb{Q}_{\ell}\right)=\mathrm{Q}-\operatorname{isom}_{k}\left(\underline{A}_{0}(\ell), \underline{A}_{0}(\ell)\right)
$$

for all $\ell$.

Proof. This follows immediately from the theorem of Tate type:

$$
\operatorname{Hom}_{k}\left(A_{1}, A_{2}\right) \otimes \mathbb{Z}_{\ell} \simeq \operatorname{Hom}_{k}\left(A_{1}\left[\ell^{\infty}\right], A_{2}\left[\ell^{\infty}\right]\right),
$$

for all $\ell$. This is due to Zarhin in the case where char $k=p \neq \ell$, due to Faltings in the case where char $k=0$, and due to de Jong in the case where char $k=p=\ell$. See [18, 4] (cf. 5]) and [2, Theorem 2.6] for more details. 
Theorem 2.2. (1) There is a natural bijection between the following two pointed sets

$$
\Lambda_{x}(k) \simeq G_{x}(\mathbb{Q}) \backslash G_{x}\left(\mathbb{A}_{f}\right) / G_{x}(\hat{\mathbb{Z}}) .
$$

Consequently, $\Lambda_{x}(k)$ is finite.

(2) Define

$$
\operatorname{mass}\left[\Lambda_{x}(k)\right]:=\sum_{\underline{A} \in \Lambda_{x}(k)} \frac{1}{\# \operatorname{Aut}_{k}(\underline{A})},
$$

where $\operatorname{Aut}_{k}(\underline{A}):=\operatorname{Isom}_{k}(\underline{A}, \underline{A})$ defined as before. Then one has mass $\left[\Lambda_{x}(k)\right]=$ $\operatorname{mass}\left[G_{x}, G_{x}(\hat{\mathbb{Z}})\right]$.

Proof. (1) Given an element $\underline{A} \in \Lambda_{x}(k)$, consider the natural map

$m(\underline{A}): \mathrm{Q}-\operatorname{isom}\left(\underline{A}, \underline{A}_{0}\right) \times \prod_{\ell} \operatorname{Isom}_{k}\left(\underline{A}_{0}(\ell), \underline{A}(\ell)\right) \rightarrow \prod_{\ell}^{\prime} \mathrm{Q}-\operatorname{isom}_{k}\left(\underline{A}_{0}(\ell), \underline{A}_{0}(\ell)\right)=G_{x}\left(\mathbb{A}_{f}\right)$

which sends $\left(\phi,\left(\alpha_{\ell}\right)_{\ell}\right)$ to $\left(\phi \alpha_{\ell}\right)_{\ell}$. If $c$ is an element in the image of $m(\underline{A})$, then the image $c(\underline{A})$ is the double coset $G_{x}(\mathbb{Q}) c G_{x}(\hat{\mathbb{Z}})$. Thus, the map $\underline{A} \mapsto c(\underline{A})$ defines a map from $\Lambda_{x}(k)$ to the double coset space $G_{x}(\mathbb{Q}) \backslash G_{x}\left(\mathbb{A}_{f}\right) / G_{x}(\hat{\mathbb{Z}})$.

Let $\underline{A}, \underline{A}^{\prime} \in \Lambda_{x}(k)$ such that $c(\underline{A})=c\left(\underline{A}^{\prime}\right)$. Write $c(\underline{A})=\left[\left(\phi \alpha_{\ell}\right)_{\ell}\right]$ and $c\left(\underline{A}^{\prime}\right)=$ $\left[\left(\phi^{\prime} \alpha_{\ell}^{\prime}\right)_{\ell}\right]$. Then there exist $b \in G_{x}(\mathbb{Q})$ and $k_{\ell} \in G_{x}\left(\mathbb{Z}_{\ell}\right)$ for all $\ell$ such that $b \phi \alpha_{\ell} k_{\ell}=$ $\phi^{\prime} \alpha_{\ell}^{\prime}$. Then

$$
(b \phi)^{-1} \phi^{\prime}=\alpha_{\ell} k_{\ell}\left(\alpha_{\ell}^{\prime}\right)^{-1} \in \mathrm{Q}-\operatorname{isom}_{k}\left(\underline{A}^{\prime}, \underline{A}\right) \cap \prod_{\ell} \operatorname{Isom}_{k}\left(\underline{A}^{\prime}(\ell), \underline{A}(\ell)\right)=\operatorname{Isom}_{k}\left(\underline{A^{\prime}}, \underline{A}\right) .
$$

Thus $\underline{A}^{\prime} \simeq \underline{A}$ and this shows the injectivity of $c$.

Given $\left[\left(\phi_{\ell}\right)_{\ell}\right]$ in $G_{x}(\mathbb{Q}) \backslash G_{x}\left(\mathbb{A}_{f}\right) / G_{x}(\hat{\mathbb{Z}})$, choose a positive integer $N$ such that $f_{\ell}:=N \phi_{\ell}^{-1}$ (from $A_{0}\left[\ell^{\infty}\right]$ to itself) is an isogeny for all $\ell$. Let $H$ be the product of the kernels of $N \phi_{\ell}^{-1}$; this is a finite subgroup scheme over $k$ invariant under the $O_{B}$-action. Take $A:=A_{0} / H$ and let $\pi: A_{0} \rightarrow A$ be the natural projection; $A$ is defined over $k$ and is equipped with a natural action by $O_{B}$ so that $\pi$ is $O_{B^{-}}$ linear. Let $\lambda \in \operatorname{Hom}\left(A, A^{t}\right) \otimes \mathbb{Q}$ be the fractional polarization on $A$ such that $\left(N^{-1} \pi\right)^{*} \lambda=\lambda_{0} ; \lambda$ is $O_{B}$-linear as $\pi$ is so. As $\pi_{\ell}$ and $f_{\ell}$ have the same kernel, there is an element $\alpha_{\ell} \in \operatorname{Isom}_{O_{B}, k}\left(\underline{A}_{0}\left[\ell^{\infty}\right], \underline{A}\left[\ell^{\infty}\right]\right)$ such that $\alpha_{\ell} f_{\ell}=\pi_{\ell}$. Then $\alpha_{\ell}=\pi_{\ell} f_{\ell}^{-1}=N^{-1} \pi_{\ell} \phi_{\ell}$ and $\alpha_{\ell}^{*} \lambda=\lambda_{0}$. This shows $\lambda \in \operatorname{Hom}_{k, O_{B}}\left(A, A^{t}\right)$ and one obtains an object $\underline{A} \in \Lambda_{x}(k)$. Put $\phi:=\left(N^{-1} \pi\right)^{-1} \in \mathrm{Q}$-isom $k\left(\underline{A}, \underline{A}_{0}\right)$. One checks

$$
\phi \alpha_{\ell}=N \pi_{\ell}^{-1} \alpha_{\ell}=N f_{\ell}^{-1}=\phi_{\ell} .
$$

This shows $c(\underline{A})=\left[\left(\phi_{\ell}\right)_{\ell}\right]$ and the surjectivity of the map $c$.

(2) It suffices to show that if $x^{\prime}=\underline{A} \in \Lambda_{x}(k)$ and $c$ any representative for the double coset $c(\underline{A})$, then $\operatorname{Aut}_{k}(\underline{A}) \simeq \Gamma_{c}$. Write $G_{x^{\prime}}$ for the group scheme over $\mathbb{Z}$ associated to $\underline{A}$ defined as in 2.1] Choose $\phi \in \mathrm{Q}$-isom ${ }_{k}\left(\underline{A}_{0}, \underline{A}\right)$ such that $\phi c_{\ell} \in$ $\operatorname{Isom}_{k}\left(\underline{A}_{0}(\ell), \underline{A}(\ell)\right)$ for all $\ell$. Note that $\alpha \in \operatorname{Aut}_{k}(\underline{A})$ if and only if $\alpha \in G_{x^{\prime}}(\mathbb{Q})$ and $\alpha_{\ell} \in \operatorname{Aut}_{k}(\underline{A}(\ell))$ for all $\ell$.

The map $\phi$ induces an isomorphism $G_{x}(\mathbb{Q}) \stackrel{\sim}{\rightarrow} G_{x^{\prime}}(\mathbb{Q})$ which sends $\beta$ to $\phi \beta \phi^{-1}=$ : $\alpha$. Note that $\alpha \in G_{x^{\prime}}(\hat{\mathbb{Z}})$ if and only if $(\phi c)^{-1} \alpha(\phi c) \in G_{x}(\hat{\mathbb{Z}})$. The latter is equivalent to $c^{-1} \beta c \in G_{x}(\hat{\mathbb{Z}})$. Therefore, the above isomorphism gives $\Gamma_{c} \simeq \operatorname{Aut}_{k}(\underline{A})$. This completes the proof. 
2.2. Let $N$ be any positive integer and $U_{N}$ be the kernel of the reduction map $G_{x}(\hat{\mathbb{Z}}) \rightarrow G_{x}(\hat{\mathbb{Z}} / N \hat{\mathbb{Z}})$. Let $\underline{A}$ be a polarized abelian $O_{B}$-variety. By an $\left(\underline{A}_{0}, U_{N}\right)$ level structure on $\underline{A}$ we mean a non-empty $U_{N}$-orbit $\bar{\eta}$ of isomorphisms $\eta$ in $\prod_{\ell} \operatorname{Isom}_{k}\left(\underline{A}_{0}(\ell), \underline{A}(\ell)\right)$. The existence of such $\bar{\eta}$ implies that the condition (i) for $\Lambda_{x}(k)$ is satisfied. Let $\bar{\eta}_{0}$ be the $U_{N}$-orbit of the identity in $\prod_{\ell} \operatorname{Isom}_{k}\left(\underline{A}_{0}(\ell), \underline{A}_{0}(\ell)\right)$. We change our notation a bit in the remaining of this section. We write $\underline{A}_{0}$ for $\left(A_{0}, \lambda_{0}, \iota_{0}, \bar{\eta}_{0}\right)$ and $\underline{A}$ for $(A, \lambda, \iota, \bar{\eta})$ in brief.

For any two $\underline{A}_{1}$ and $\underline{A}_{2}$ over a field $k$, denote by

- Q-isom ${ }_{k}\left(\underline{A}_{1}, \underline{A}_{2}\right)$ and Q-isom $k\left(\underline{A}_{1}(\ell), \underline{A}_{2}(\ell)\right)$ the sets which have the same meaning as in (2.1);

- $\operatorname{Isom}_{k}\left(\underline{A}_{1}, \underline{A}_{2}\right)$ the set of elements $\varphi$ in $\operatorname{Isom}_{k}\left(\left(A_{1}, \lambda_{1}, \iota_{1}\right),\left(A_{2}, \lambda_{2}, \iota_{2}\right)\right)$ satisfying $\varphi_{*} \bar{\eta}_{1}=\bar{\eta}_{2}$

- $\operatorname{Isom}_{k}\left(\underline{A}_{1}(\ell), \underline{A}_{2}(\ell)\right)$ the set of elements $\varphi$ in $\operatorname{Isom}_{k}\left(\left(A_{1}, \lambda_{1}, \iota_{1}\right)(\ell),\left(A_{2}, \lambda_{2}, \iota_{2}\right)(\ell)\right)$ satisfying $\varphi_{*} \bar{\eta}_{1, \ell}=\bar{\eta}_{2, \ell}$.

Let $\Lambda_{x, N}(k)$ denote the set of isomorphism classes of polarized abelian $O_{B^{-}}$ varieties with an $\left(\underline{A}_{0}, U_{N}\right)$-level structure $(A, \lambda, \iota, \bar{\eta})$ over $k$ such that Q-isom ${ }_{k}\left(\underline{A}_{0}, \underline{A}\right) \neq$ $\emptyset$. The same proof of Theorem 2.2 without modification gives the following variant.

Theorem 2.3. There is a natural bijection

$$
\Lambda_{x, N}(k) \simeq G_{x}(\mathbb{Q}) \backslash G_{x}\left(\mathbb{A}_{f}\right) / U_{N} .
$$

Furthermore, one has mass $\left[\Lambda_{x . N}(k)\right]=\operatorname{mass}\left(G_{x}, U_{N}\right)$.

Lemma 2.4. If $N \geq 3$, then the group $\operatorname{Aut}_{k}(\underline{A})$ is trivial for any object $\underline{A}=$ $(A, \lambda, \iota, \bar{\eta})$ in $\Lambda_{x, N}(k)$.

Proof. Clearly $\operatorname{Aut}_{k}(\underline{A}) \subset \operatorname{Aut}_{k}(A, \lambda, \bar{\eta})$. By a theorem of Serre, we have $\operatorname{Aut}_{k}(A, \lambda, \bar{\eta})=\{1\}$. Therefore, $\operatorname{Aut}_{k}(\underline{A})$ is trivial.

Remark 2.5. When the ground $k$ has characteristic $p$, the usual assumption of $O_{B}$ for good reduction is not required in Theorems 2.2 and 2.3

\section{Abelian VARIETIES of ARITHMEtic type}

In this section we define a class of abelian varieties with additional structures those share the similar properties as supersingular abelian varieties. We call them of arithmetic type (or of $B$-arithmetic type to be precise) as those have rich arithmetic properties. The naive definition is given in Def. 3.1. After studying basic properties of these abelian varieties, we give another equivalent but refined definition in Def. 3.8 .

3.1. In the following, polarized $O_{B}$-abelian varieties will be assumed implicitly to have the properties: $(*)$ There is a non-degenerate $\mathbb{Q}$-valued skew-Hermitian $B$-space $(V, \psi)$ such that $2 \operatorname{dim} A=\operatorname{dim}_{\mathbb{Q}} V$.

That is, $V$ is a left faithful finite $B$-module, and $\psi: V \times V \rightarrow \mathbb{Q}$ is a nondegenerate alternating pairing on $V$ such that $\psi(b x, y)=\psi\left(x, b^{*} y\right)$ for all $b \in B$ and $x, y, \in V$. In other words, the objects $\underline{A}$ we consider are those that can occur in the Shimura variety attached to the reductive group defined by a PEL datum $(B, *, V, \psi)$. However, the moduli space of PEL-type itself will not be specified explicitly here. 
Definition 3.1. Let $k$ be a field of finite type over its prime field. Let $\underline{A}=(A, \lambda, \iota)$ be a polarized abelian $O_{B}$-variety over $k$ such that $\operatorname{End}\left(A_{\bar{k}}\right)=\operatorname{End}\left(A_{k}\right)$. Let $T_{\ell}:=T_{\ell}(A)$ denote the Tate module of $A(\ell \neq \operatorname{char} k)$. Write $\operatorname{GAut}_{B}\left(T_{\ell}\right)$ or $\operatorname{GAut}_{B}\left(T_{\ell},\langle\rangle,\right)$ for the group

$$
\left\{g \in \operatorname{End}_{B_{\ell}}\left(V_{\ell}\right) ; g^{\prime} g \in \mathbb{Q}_{\ell}^{\times}\right\},
$$

where $g \mapsto g^{\prime}$ is the involution induced by $\lambda$. Let $\rho_{\ell}: \mathcal{G}_{k}:=\operatorname{Gal}\left(k_{s} / k\right) \rightarrow$ $\operatorname{GAut}_{B}\left(T_{\ell}\right)$ be the associated $\ell$-adic Galois representation. We call $\underline{A}$ over $k$ is of arithmetic type if the image $\rho_{\ell}\left(\mathcal{G}_{k}\right)$ is contained in the center of $\operatorname{GAut}_{B}\left(T_{\ell}\right)$ for all $\ell \neq \operatorname{char} k$.

We write $B_{\ell}:=B \otimes \mathbb{Q}_{\ell}$ and $V_{\ell}=T_{\ell} \otimes \mathbb{Q}_{\ell}$. Let $G_{\ell}:=\rho_{\ell}\left(\mathcal{G}_{k}\right)$, the image of the $\ell$-adic Galois representation, and let $G_{\ell}^{\text {alg }}$ be the algebraic envelope of $G_{\ell}$, the smallest algebraic subgroup $H$ of $\mathrm{GL}\left(V_{\ell}\right)$ over $\mathbb{Q}_{\ell}$ such that $H\left(\mathbb{Q}_{\ell}\right)$ contains $G_{\ell}$. Write $G^{0}$ for the neutral component of an algebraic group $G$.

Lemma 3.2. Let $\underline{A}=(A, \lambda, \iota)$ be a polarized abelian $O_{B}$-variety over $k$ as in Def. 3.1.

(1) If $O_{B}=\mathbb{Z}$, that is, $\underline{A}=(A, \lambda)$ be a polarized abelian variety, then $\underline{A}$ is of arithmetic type if and only if char $k=p>0$ and $A$ is supersingular.

(2) If $B$ is a commutative semi-simple algebra and $[B: \mathbb{Q}]=2 \operatorname{dim} A$, that is, $A$ has complex multiplication by $B$, then $\underline{A}$ is of arithmetic type.

(3) Let $\underline{A}^{\prime}$ is another polarized abelian $O_{B}$-variety such that there is an $O_{B}$-linear isogeny $\varphi: A \rightarrow A^{\prime}$ which preserves the polarizations. Then $\underline{A}$ is of arithmetic type if and only if $\underline{A}^{\prime}$ is also of arithmetic type.

(4) Let $B$ be a semi-simple subalgebra of a matrix algebra $M_{n}(K)$, and let $C_{B}$ be the commutant of $B$ in $M_{n}(K)$. Then the center $Z\left(C_{B}\right)$ of $C_{B}$ of $B$ is the center $Z(B)$ of $B$.

Proof. (1) If the image of the Galois group lies in the center of $\operatorname{GSp}_{2 g}\left(\mathbb{Z}_{\ell}\right)$, which consists of the scalar matrices, then one has $\operatorname{dim} \operatorname{End}^{0}(A)=4 g^{2}$. This is possible only when char $k=p>0$ and $A$ is supersingular.

(2) Since $\operatorname{End}_{O_{B}}\left(T_{\ell}(A)\right)$ is already commutative, the statement follows.

(3) This is clear.

(4) By the bi-commutant theorem, the elements of $M_{n}(K)$ which commute with $C_{B}$ lie in $B$. Therefore, $Z\left(C_{B}\right)$ consists of elements in $B$ commuting with $B$. This shows $Z\left(C_{B}\right)=Z(B)$.

3.2. Let $\underline{A}=(A, \lambda, \iota)$ is a polarized abelian $O_{B}$-variety over $k$ as in Def. 3.1. Write the semi-simple algebra $B$ into simple factors $\oplus_{i=1}^{r} M_{n_{i}}\left(D_{i}\right)$, where $D_{i}$ is a division algebra over $\mathbb{Q}$ with a positive involution $*_{i}$. According to this decomposition the abelian variety $A$ is isogenous to $\prod A_{i}^{n_{i}}$. One has ring monomorphisms $\iota_{i}: D_{i} \rightarrow$ $\operatorname{End}^{0}\left(A_{i}\right):=\operatorname{End}\left(A_{i}\right) \otimes \mathbb{Q}$. Write $V_{i}$ for $T_{\ell}\left(A_{i}\right) \otimes \mathbb{Q}_{\ell}$ and one has

$$
\begin{gathered}
V_{\ell}=\prod_{i=1}^{r} V_{i}^{\oplus n_{i}}, \\
\operatorname{End}_{B}\left(V_{\ell}\right)=\oplus_{i=1}^{r} \operatorname{End}_{D_{i}}\left(V_{i}\right) .
\end{gathered}
$$


Let $g \mapsto g^{\prime}$ be the adjoint with respect to the alternating pairing $\langle$,$\rangle on V_{\ell}$. Then $\operatorname{GAut}_{B}\left(V_{\ell},\langle\rangle,\right)$ consists of elements $g=\left(g_{i}\right) \in \prod \operatorname{End}_{D_{i}}\left(V_{i}\right)$ such that $g_{1}^{\prime} g_{1}=$ $g_{2}^{\prime} g_{2}=\cdots=g_{r}^{\prime} g_{r} \in \mathbb{Q}_{\ell}^{\times}$.

We have projections $p_{i}: \operatorname{GAut}_{B}\left(V_{\ell},\langle\rangle,\right) \rightarrow \operatorname{GAut}_{D_{i}}\left(V_{i},\langle,\rangle_{i}\right)$ and these induce $p_{i}: Z\left(\operatorname{GAut}_{B}\left(V_{\ell},\langle\rangle,\right)\right) \rightarrow Z\left(\operatorname{GAut}_{D_{i}}\left(V_{i},\langle,\rangle_{i}\right)\right)$. If $\rho_{i, \ell}$ is the $\ell$-adic Galois representation attached to $\underline{A}_{i}$, then one has $p_{i} \circ \rho_{\ell}=\rho_{i, \ell}$. This shows that if $\underline{A}$ is of arithmetic type, then so as each factor $\underline{A}_{i}$. The converse is also true as $Z\left(\operatorname{GAut}_{B}\left(V_{\ell},\langle\rangle,\right)\right) \hookrightarrow \prod_{i} Z\left(\operatorname{GAut}_{D_{i}}\left(V_{i},\langle,\rangle_{i}\right)\right)$. Thus one has

3.2.1. Notation as above. Then $\underline{A}$ is of arithmetic type if and only if each factor $\underline{A}_{i}$ is of arithmetic type.

Definition 3.3. Let $(B, *)$ be as in 2.1 and assume that $B$ is a division algebra. A polarized abelian $O_{B}$-variety is said to be of type (D \& 0-dim) if $B$ is of type (III) in the Albert classification and $2 \operatorname{dim} A=[B: \mathbb{Q}]$.

Recall [10, Section 23] that $B$ is of type (III) if $B$ is a totally definite quaternion algebra over a totally real number field $F$ and $*$ is the main involution, the unique positive involution.

Lemma 3.4. Assume that $B$ is a division algebra. Let $\underline{A}$ be a polarized abelian $O_{B}$-variety of type (D \& 0-dim). Then

(1) $T_{\ell}$ is a free $O_{F} \otimes \mathbb{Z}_{\ell}$-module of rank 4.

(2) $V_{\ell}$ is a free $B_{\ell}$-module of rank 1.

(3) $\operatorname{GAut}_{B_{\ell}}\left(V_{\ell},\langle\rangle,\right)$ is an extension of a normal commutative subgroup by a finite 2-torsion group.

(4) The center $Z\left(\operatorname{GAut}_{B_{\ell}}\left(V_{\ell},\langle\rangle,\right)\right)$ consists of elements a in $F_{\ell}:=F \otimes \mathbb{Q}_{\ell}$ with $a^{2} \in \mathbb{Q}_{\ell}^{\times}$.

Proof. The statement (1) follows from the fact that $\operatorname{Tr}\left(a ; V_{\ell} / \mathbb{Q}_{\ell}\right)=4 \operatorname{Tr}_{F / \mathbb{Q}}(a)$ for all $a \in O_{F}$. The statement (2) follows from (1). To show the statement (3), we regard $G:=\operatorname{GAut}_{B}\left(V_{\ell},\langle\rangle,\right)$ as an algebraic group over $\mathbb{Q}_{\ell}$ and show that its neutral component $G^{0}$ is a torus.

Let $V_{\ell}=B_{\ell}$ as a left $B_{\ell}$-module. Let $():, B_{\ell} \times B_{\ell} \rightarrow B_{\ell}$ be the lifting of $\langle$,$\rangle . One has \langle x, y\rangle=\operatorname{Trd}_{B_{\ell} / \mathbb{Q}_{\ell}}\left(x \alpha y^{*}\right)$, where $\alpha=(1,1)$ and $\alpha^{*}=-\alpha$. Any element in $\operatorname{End}_{B_{\ell}}\left(V_{\ell}\right)$ is a right translation $\rho_{g}$ for some $g \in B_{\ell}$. The condition $\langle x g, y g\rangle=c(g)\langle x, y\rangle$ gives $\operatorname{Trd}_{B_{\ell} / \mathbb{Q}_{\ell}}\left(x g \alpha g^{*} y^{*}\right)=\operatorname{Trd}_{B_{\ell} / \mathbb{Q}_{\ell}}\left(x c(g) \alpha y^{*}\right)$. Therefore, the group $G$ is the subgroup of $B_{\ell}^{\text {opp }, \times}$ defined by the relation $g \alpha g^{*}=c(g) \alpha$ for some $c(g) \in \mathbb{Q}_{\ell}^{\times}$. Choose the isomorphism $B_{\ell}^{\text {opp }} \simeq B_{\ell}$ which sends $g \mapsto g^{-1}$; the group $G$ is identified with the subgroup of $B_{\ell}^{\times}$defined by the same relation.

For each $\sigma \in \Sigma:=\operatorname{Hom}\left(F_{\ell}, \overline{\mathbb{Q}}_{\ell}\right)$, put $B_{\sigma}=B_{\ell} \otimes_{F_{\ell}, \sigma} \overline{\mathbb{Q}}_{\ell} \simeq M_{2}\left(\overline{\mathbb{Q}}_{\ell}\right)$. Let $j=$ $\left(\begin{array}{cc}0 & -1 \\ 1 & 0\end{array}\right)$ and $g \in B_{\sigma}$, one computes

$$
j g^{*} j^{-1}=\left(\begin{array}{cc}
0 & -1 \\
1 & 0
\end{array}\right)\left(\begin{array}{cc}
d & -b \\
-c & a
\end{array}\right)\left(\begin{array}{cc}
0 & 1 \\
-1 & 0
\end{array}\right)=\left(\begin{array}{ll}
a & c \\
b & d
\end{array}\right)=g^{t} .
$$

Write $\alpha=\beta j$, then $\beta^{t}=j \beta^{*} j^{-1}=-\alpha^{*} j^{-1}=\beta$ and the relation defining $G$ becomes $g \beta g^{t}=c(g) \beta$ for some $c(g)$. We have proved

$G_{\overline{\mathbb{Q}}_{\ell}} \simeq\left\{\left(g_{\sigma}\right) \in \mathrm{GL}_{2}^{\Sigma} ; g_{\sigma} g_{\sigma}^{t}=c\right.$ for some $c \in \overline{\mathbb{Q}}_{\ell}^{\times}$(independent of $\sigma$ ), $\left.\forall \sigma \in \Sigma\right\}$, and 
$G_{\overline{\mathbb{Q}}_{\ell}}^{0} \simeq\left\{\left(\begin{array}{cc}a_{i} & b_{i} \\ -b_{i} & a_{i}\end{array}\right) \in \mathrm{GL}_{2}^{d} ; a_{i}^{2}+b_{i}^{2}=c\right.$ for some $\left.c \in \overline{\mathbb{Q}}_{\ell}^{\times}, \forall 1 \leq i \leq d=[F: \mathbb{Q}]\right\}$.

This shows that $G^{0}$ is a torus.

(4) This follows directly from the computation in (3).

Lemma 3.5. For any polarized abelian $O_{B}$-variety $\underline{A}$, the center $Z\left(\operatorname{GAut}_{B}\left(V_{\ell},\langle\rangle,\right)\right)$ consists of elements $a \in Z(B) \otimes \mathbb{Q}_{\ell}$ such that $a^{*} a \in \mathbb{Q}_{\ell}^{\times}$, where $\mathbb{Q}_{\ell}$ is included in $Z(B) \otimes \mathbb{Q}_{\ell}$ diagonally.

Proof. By the argument (3.2) one reduces to the case where $B$ is a division algebra. The case of type (D \& 0 -dim) has been treated in Lemma 3.4 Now suppose that $\underline{A}$ is not of type (D \& 0 -dim).

For an algebra $E$ and a subset $G$ of $E$, we write

$$
Z(E, G):=\{x \in E ; g x=x g \forall g \in G\} .
$$

Let $E:=\operatorname{End}_{B}\left(V_{\ell}\right)$ and $G$ be the algebraic group over $\mathbb{Q}_{\ell}$ defined by $\underline{V}_{\ell}$; we have particularly $G\left(\mathbb{Q}_{\ell}\right)=\operatorname{GAut}_{B}\left(V_{\ell},\langle\rangle,\right)$. It then suffices to show that $Z(B) \otimes$ $\mathbb{Q}_{\ell}=Z\left(E, G\left(\mathbb{Q}_{\ell}\right)\right)$. We have $Z(B) \otimes \mathbb{Q}_{\ell} \subset Z\left(E, G\left(\mathbb{Q}_{\ell}\right)\right)$. So it suffices to show $\operatorname{dim}_{\mathbb{Q}_{\ell}} Z(B) \otimes \mathbb{Q}_{\ell}=\operatorname{dim}_{\mathbb{Q}_{\ell}} Z\left(E, G\left(\mathbb{Q}_{\ell}\right)\right)$. Since $\operatorname{dim}_{\mathbb{Q}_{\ell}} Z(B) \otimes \mathbb{Q}_{\ell}=\operatorname{dim}_{\overline{\mathbb{Q}}_{\ell}} Z(B) \otimes \overline{\mathbb{Q}}_{\ell}$ and

$$
\operatorname{dim}_{\overline{\mathbb{Q}}_{\ell}} Z\left(E \otimes \overline{\mathbb{Q}}_{\ell}, G^{0}\left(\overline{\mathbb{Q}}_{\ell}\right)\right)=\operatorname{dim}_{\mathbb{Q}_{\ell}} Z\left(E, G^{0}\left(\mathbb{Q}_{\ell}\right)\right) \geq \operatorname{dim}_{\mathbb{Q}_{\ell}} Z\left(E, G\left(\mathbb{Q}_{\ell}\right)\right)
$$

(as $G^{0}\left(\mathbb{Q}_{\ell}\right)$ is Zariski dense in $\left.G^{0}\right)$, it suffices to show that $\operatorname{dim}_{\overline{\mathbb{Q}}_{\ell}} Z(B) \otimes \overline{\mathbb{Q}}_{\ell}=$ $\operatorname{dim}_{\overline{\mathbb{Q}}_{\ell}} Z\left(E \otimes \overline{\mathbb{Q}}_{\ell}, G^{0}\left(\overline{\mathbb{Q}}_{\ell}\right)\right)$. Decomposing into simple factors, we have three cases:

(a) $E=M_{n}\left(\overline{\mathbb{Q}}_{\ell}\right) \times M_{n}\left(\overline{\mathbb{Q}}_{\ell}\right), *:(A, B) \mapsto\left(B^{t}, A^{t}\right)$ and $G=G U_{n}$.

(b) $E=M_{2 n}\left(\overline{\mathbb{Q}}_{\ell}\right), *$ is the standard symplectic involution, and $G=G S p_{2 n}$.

(c) $E=M_{2 n}\left(\overline{\mathbb{Q}}_{\ell}\right), *: A \mapsto A^{t}$ and $G=G O_{2 n}(n \geq 2)$.

Then we have the cases (a) $Z\left(E, G^{0}\right)=\left\{\left(a I_{n}, b I_{n}\right) ; a, b \in \overline{\mathbb{Q}}_{\ell}\right\}$; (b) $Z\left(E, G^{0}\right)=$ $\left\{a I_{2 n} ; a \in \overline{\mathbb{Q}}_{\ell}\right\} ;$ (c) $Z\left(E, G^{0}\right)=\left\{a I_{2 n} ; a \in \overline{\mathbb{Q}}_{\ell}\right\}$. From this one sees that $\operatorname{dim}_{\overline{\mathbb{Q}}_{\ell}} Z(B) \otimes$ $\overline{\mathbb{Q}}_{\ell}=\operatorname{dim}_{\overline{\mathbb{Q}}_{\ell}} Z\left(E \otimes \overline{\mathbb{Q}}_{\ell}, G^{0}\left(\overline{\mathbb{Q}}_{\ell}\right)\right)$. This finishes the proof.

Lemma 3.6. Let $\underline{A}=(A, \lambda, \iota)$ be a polarized abelian $O_{B}$-variety and $\lambda^{\prime}$ be another $O_{B}$-linear polarization. Then $\left(A, \lambda^{\prime}, \iota\right)$ is of arithmetic type if and only if $\underline{A}$ is of arithmetic type.

Proof. By Lemma 3.5, the center of $\operatorname{GAut}_{B}\left(V_{\ell},\langle\rangle,\right)$ is independent of the choice of polarizations. Therefore, the assertion is proved.

Lemma 3.7. Let $k_{0}$ be a field of finite type over its prime field and $k$ be a field finitely generated over $k_{0}$. Let $\underline{A}$ be a polarized abelian $O_{B}$-variety over $k_{0}$. If $\underline{A}$ is of arithmetic type then so as $\underline{A}_{k}:=\underline{A} \otimes_{k_{0}} k$. Conversely, if $\underline{A}_{k}$ is of arithmetic type, then so as $\underline{A}_{k^{\prime}}$ for a finite extension $k^{\prime}$ of $k_{0}$.

Proof. Let $k_{s}$ be a separable closure of $k$ and $k_{0, s}$ the algebraic closure of $k_{0}$ in $k_{s}$. Let $k_{1}$ be the algebraic closure of $k_{0}$ in $k$. The restriction gives a surjective homomorphism $r: \mathcal{G}_{k} \rightarrow \mathcal{G}_{k_{1}}$ of Galois groups. We also have the Galois equivariant 
isomorphism $s: A\left[\ell^{n}\right]\left(k_{0, s}\right) \simeq A\left[\ell^{n}\right]\left(k_{s}\right)$ in the sense that $r(\sigma) x=\sigma(s(x))$ for $x \in A\left[\ell^{n}\right]\left(k_{0, s}\right)$ and $\sigma \in \mathcal{G}_{k}$. This gives rise to the commutative diagram

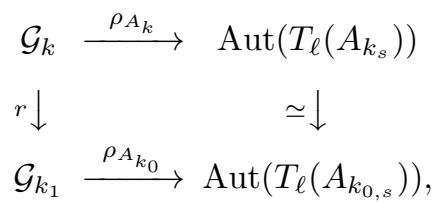

and one has $\rho_{A_{k}}\left(\mathcal{G}_{k}\right)=\rho_{A_{k_{0}}}\left(\mathcal{G}_{k_{1}}\right)$. It follows that $\underline{A}_{k}$ is of arithmetic if and only if $\underline{A}_{k_{1}}$ is so. From this the first and second statement follow.

Lemma 3.2 (3) and Lemmas 3.6 and 3.7 suggest the following alternative definition, which is more refined.

Definition 3.8. Let $(B, *)$ be as in 2.1 and $(A, \iota)$ be an abelian $B$-variety up to isogeny over $k$ as in Def. 3.1. The pair $(A, \iota)$ is said to be of $B$-arithmetic type or simply of arithmetic type if there is a finite extension $k^{\prime} / k$ such that $\rho_{\ell}\left(\mathcal{G}_{k^{\prime}}\right)$ lies in the center of $\operatorname{GAut}_{B}\left(V_{\ell}\right)$ for some $B$-linear polarization $\lambda$ and for one $\ell \neq$ char $k$. An abelian $B$-variety up to isogeny is said to be of arithmetic type if it is so over a field of finite type over its prime field.

For any abelian $B$-variety $(A, \iota)$ up to isogeny, there always exists a $B$-linear polarization on $(A, \iota)$ (see [9, Section 9]). We now show the $\ell$-independence of $G_{\ell}^{\text {alg }}$ for abelian varieties of arithmetic type.

Lemma 3.9. Let $\underline{A}=(A, \iota)$ be an abelian B-variety up to isogeny. If $\underline{A}$ is of arithmetic type, then $A$ is of CM type.

Proof. Since $\underline{A}$ is of arithmetic type, $G_{\ell}$ is commutative. Denote by $\mathbb{Q}_{\ell}\left[G_{\ell}\right]$ the subalgebra of $\operatorname{End}\left(V_{\ell}\right)$ generated by $G_{\ell}$. By the semi-simplicity of Tate modules due to Faltings and Zarhin [4, 18, $\mathbb{Q}_{\ell}\left[G_{\ell}\right]$ is a (commutative) semi-simple subalgebra. Let $L$ be a maximal semi-simple commutative subalgebra in $\operatorname{End}^{0}(A)$, then $L \otimes \mathbb{Q}_{\ell}$ is a maximal commutative semi-simple algebra in $\operatorname{End}^{0}(A) \otimes \mathbb{Q}_{\ell}$. By the theorem of Faltings and Zarhin on Tate's conjecture loc. cit., we have $\operatorname{End}^{0}(A) \otimes \mathbb{Q}_{\ell}=\operatorname{End}_{\mathbb{Q}_{\ell}\left[G_{\ell}\right]}\left(V_{\ell}\right)$. Hence $L \otimes \mathbb{Q}_{\ell}$ becomes a maximal semi-simple commutative subalgebra in $\operatorname{End}_{\mathbb{Q}_{\ell}\left[G_{\ell}\right]}\left(V_{\ell}\right)$. Since $\mathbb{Q}_{\ell}\left[G_{\ell}\right]$ is commutative and semisimple, any maximal semi-simple commutative subalgebra in $\operatorname{End}_{\mathbb{Q}_{\ell}\left[G_{\ell}\right]}\left(V_{\ell}\right)$ has degree $2 g$ over $\mathbb{Q}_{\ell}$. This shows $[L: \mathbb{Q}]=2 g$ and the proof is complete.

Proposition 3.10. Let $(A, \iota)$ be an abelian B-variety of arithmetic type over $k$ as in Def. 3.8. Then the algebraic envelope $G_{\ell}^{\text {alg }}$ is independent of $\ell$ for all $\ell \neq$ char $k$. That is, there is a $\mathbb{Q}$-subgroup $G$ of $\mathrm{GL}_{2 g}$ such that $G \otimes \mathbb{Q}_{\ell} \simeq G_{\ell}^{\text {alg }}$ for all $\ell \neq$ char $k$.

Proof. By Lemma 3.9, $A$ is of CM-type. The semi-simple part of $G_{\ell}^{\text {alg }}$ is trivial. By Bogomolov's theorem, the neutral component $\left(G_{\ell}^{\text {alg }}\right)^{0}$ is independent of $\ell$ (see [15. 2.2.5], also see the remark in 2.3 of loc. cit. for the function field case). By a theorem of Serre [15] that the component group $G_{\ell}^{\text {alg }} /\left(G_{\ell}^{\text {alg }}\right)^{0}$ is independent of $\ell$, one shows that $G_{\ell}^{\text {alg }}$ is independent of $\ell$.

Remark 3.11. In 3.2 - Proposition 3.10 we have shown that $\underline{A}$ is of arithmetic type in the sense of Def. 3.1 if and only if its underlying abelian $O_{B}$-variety $(A, \iota)$ is of 
arithmetic type in the sense of Def. 3.8. According to Definition 3.8 the property for an object $\underline{A}$ being of arithmetic type is independent of the ground field for which it is defined.

Theorem 3.12. Let $k$ be an algebraically closed field and let $x=\underline{A}$ be a polarized abelian $O_{B}$-variety over $k$. Suppose that $\underline{A}$ is of arithmetic type.

(1) $G_{x}\left(\mathbb{Z}_{\ell}\right)=\operatorname{Isom}_{k}(\underline{A}(\ell))$ for all $\ell$.

(2) There is a natural bijection

$$
\Lambda_{x, N}(k) \simeq G_{x}(\mathbb{Q}) \backslash G_{x}\left(\mathbb{A}_{f}\right) / U_{N} .
$$

(3) $\operatorname{mass}\left[\Lambda_{x . N}(k)\right]=\operatorname{mass}\left(G_{x}, U_{N}\right)$.

Proof. The statements (2) and (3) follow from the statement (1) and Theorems 2.2 and 2.3 . We prove (1). Let $k_{0}$ be a finitely generated field for which $\underline{A}$ is defined and we may assume that $k$ is an algebraic closure of $k_{0}$.

If char $k=0$, then $G_{\ell}:=\rho_{\ell}\left(\mathcal{G}_{k_{0}}\right)$ is in $Z\left(\operatorname{GAut}_{B}\left(V_{\ell}\right)\right)$ for all $\ell$. By Faltings' theorem [4], one has

$$
G_{x}\left(\mathbb{Z}_{\ell}\right)=\operatorname{Aut}_{k_{0}}(\underline{A}(\ell))=Z\left(\operatorname{Aut}_{k}(\underline{A}(\ell)), G_{\ell}\right) .
$$

It follows that $G_{x}\left(\mathbb{Z}_{\ell}\right)=\operatorname{Aut}_{k}(\underline{A}(\ell))$. This proves the case of characteristic zero.

If char $k=p>0$, then replacing $\underline{A}$ by an isogeny we may assume that $k_{0}$ is a finite field, as $A$ is of CM-type (Lemma 3.9 and a theorem of Grothendieck [10, p. 220]). Let Frob be the geometric Frobenius in $\mathcal{G}_{k_{0}}$ and $\pi_{A}$ the relative Frobenius endomorphism on $A$. By the $p$-adic version of Tate's theorem on endomorphisms over finite fields, it then suffices to show that $\pi_{A}$ lies in $Z(B) \otimes \mathbb{Q}_{p}$, the center of $\operatorname{End}_{B}^{0}\left(A\left[p^{\infty}\right]\right)$. Since $A$ is of arithmetic type, one has $\pi_{A}=\rho_{\ell}(\operatorname{Frob}) \in Z(B) \otimes \mathbb{Q}_{\ell}$ (Lemma 3.5). Consider $\mathbb{Q}\left[\pi_{A}\right]$ and $Z(B)$ as linear subspaces of $\operatorname{End}^{0}(A)$; then $\mathbb{Q}\left[\pi_{A}\right]=\mathbb{Q}_{\ell}\left[\pi_{A}\right] \cap \operatorname{End}^{0}(A) \subset Z(B) \otimes \mathbb{Q}_{\ell} \cap \operatorname{End}^{0}(A)=Z(B)$. Therefore, $\pi_{A} \in Z(B) ;$ this proves the statement (1).

\section{Classification}

In this section we classify abelian $B$-varieties of arithmetic type up to isogeny. Due to Lemma 3.9. it suffices to classify these objects which are defined either over a number field or over a finite field. It is enough to consider the case where $B$ is a division algebra by 3.2.1. From Lemma 4.1 to Proposition 4.2, we assume that $B$ is a division algebra.

Let $\mathbb{P}$ be a prime field, $k$ be an algebraic closure of $\mathbb{P}$ and $k_{0}$ be a finite extension of $\mathbb{P}$ in $k$. Let $\underline{A}$ be an abelian $O_{B}$-variety over $k_{0}$.

Lemma 4.1. If the positive involution $*$ on $B$ is of first kind, then $\underline{A}$ is of arithmetic type if and only if char $k=p>0$ and $A$ is supersingular.

Proof. If $\underline{A}$ is of arithmetic type, then by Lemma 3.5 $G_{\ell}$ is contained in $\mathbb{Q}_{\ell}^{\times}$ after replacing $k_{0}$ by a finite extension. Then $\operatorname{End}^{0}(A)$ has dimension $4 g^{2}$ by Tate's theorem. This implies char $k=p>0$ and $A$ is supersingular. The other implication is obvious.

4.1. Lemma 4.1 classifies the abelian varieties of $B$-arithmetic type in the case of first kind. Suppose that $*$ is of second kind. Let $K$ be the center of $B$ and $F$ be the maximal totally real subfield of $K$. 
4.1.1. Let $\iota_{0}: O_{K} \rightarrow \operatorname{End}(A)$ be the restriction of $\iota$. Then $(A, \iota)$ is of $B$-arithmetic type if and only if $\left(A, \iota_{0}\right)$ is of $K$-arithmetic type. Indeed, it follows from Lemma 3.5 that the centers $Z\left(\operatorname{GAut}_{B}\left(V_{\ell},\langle\rangle,\right)\right)$ and $Z\left(\operatorname{GAut}_{K}\left(V_{\ell},\langle\rangle,\right)\right)$ are the same. Therefore, the classification is reduced to the case where $B$ is a CM field $K$.

4.1.2. The abelian variety $A$ is isogenous to $\prod_{j=1}^{r} A_{j}^{n_{j}}$, denoted by $A \sim \prod_{j=1}^{r} A_{j}^{n_{j}}$, where each $A_{j}$ is a simple abelian variety and $A_{i}$ is not isogenous to $A_{j}$ for $i \neq j$. If $\underline{A}$ is of $K$-arithmetic type, then we have

$$
\operatorname{End}_{K}^{0}(A) \otimes \mathbb{Q}_{\ell} \simeq \operatorname{End}_{K_{\ell}}\left(V_{\ell}\right) .
$$

Note that $V_{\ell}$ is a free $K_{\ell}$-module. The right hand side is isomorphic to $M_{n}\left(K_{\ell}\right)$ and has $\mathbb{Q}_{\ell}$-dimension $n^{2} d$, where $[K: \mathbb{Q}]=d$ and $2 g=d n$. Put $B_{j}:=A_{j}^{n_{j}}$, $b_{j}=\operatorname{dim} B_{j}$ and let $m_{j}:=2 b_{j} / d$. We have

$$
\operatorname{dim}_{\mathbb{Q}} \operatorname{End}_{K}^{0}\left(B_{j}\right) \leq \operatorname{dim}_{\mathbb{Q}_{\ell}} \operatorname{End}_{K_{\ell}}\left(V_{\ell}\left(B_{j}\right)\right)=d m_{j}^{2} .
$$

We also have $\operatorname{End}_{K}^{0}(A)=\prod_{j} \operatorname{End}_{K}^{0}\left(B_{j}\right)$. From the dimensions of the abelian varieties $B_{j}$ and those of their endomorphism algebras, we have

$$
\sum_{j=1}^{r} m_{j}=n, \quad n^{2} \leq \sum_{j=1}^{r} m_{j}^{2} .
$$

This shows $r=1$. We have shown that if $\underline{A}$ is of arithmetic type then it is isogenous to a self product of a simple factor.

Proposition 4.2. Suppose that char $k=0$. Let $\underline{A}=(A, \iota)$ be an abelian $O_{K}$ variety with a $C M$ field $K$. Then $\underline{A}$ is of $K$-arithmetic type if and only if $A \sim A_{1}^{n}$, where $A_{1}$ is a simple abelian variety with $C M$ by a CM field $K_{1}$, and the image of the homomorphism $\iota: K \rightarrow \operatorname{End}^{0}(A)=M_{n}\left(K_{1}\right)$ contains the center $K_{1}$.

Proof. $\quad(\Rightarrow)$ Suppose that $A$ is of $K$-arithmetic type. The first statement is proved in 4.1.2. We regard $K$ as a subfield of $\operatorname{End}^{0}(A)$ via $\iota$. Let $\widetilde{K}$ the composite of $K$ and $K_{1}$. We want to show that $K=\widetilde{K}$. The centralizer of $K$ in $M_{n}\left(K_{1}\right)$, same as that of $\widetilde{K}$, has $\mathbb{Q}$-dimension

$$
[\widetilde{K}: \mathbb{Q}](\operatorname{dim} V /[\widetilde{K}: \mathbb{Q}])^{2}=4 g^{2} /[\widetilde{K}: \mathbb{Q}] .
$$

While $\operatorname{End}_{K_{\ell}}\left(V_{\ell}\right)$ has $\mathbb{Q}_{\ell}$-dimension $4 g^{2} /\left[K_{\ell}: \mathbb{Q}_{\ell}\right]$. It follows that $[\widetilde{K}: \mathbb{Q}]=[K: \mathbb{Q}]$ and $K \supset K_{1}$.

$(\Leftarrow)$ First, one has $G_{\ell} \subset \operatorname{End}_{\operatorname{End}^{0}(A)}\left(V_{\ell}\right)$. Since $n\left[K_{1}: \mathbb{Q}\right]=\operatorname{dim}_{\mathbb{Q}_{\ell}} V_{\ell}$, the commutant $\operatorname{End}_{\operatorname{End}^{0}(A)}\left(V_{\ell}\right)$ is $K_{1, \ell}$. The center of $\operatorname{End}_{K_{1}}\left(V_{\ell}\right)$ is $K_{1, \ell}$. So the Galois group $G_{\ell}$ is contained in the center of $\operatorname{End}_{K_{1}}\left(V_{\ell}\right)$. This shows that $A$ is of $K_{1}$ arithmetic type, particularly of $K$-arithmetic type.

Thereafter, the characteristic of $k$ will be $p>0$. We recall the definition of basic abelian varieties with additional structures in the sense of Kottwitz (cf. [8] and [14, p. 291, 6.25]).

Definition 4.3. Let $W$ be the ring of Witt vectors over $k$ and $L$ be the fraction field of $W$. Let $(B, *)$ remain as in 2.1.

(1) Let $\left(V_{p}, \psi_{p}\right)$ be a $\mathbb{Q}_{p}$-valued non-degenerate skew-Hermitian $B_{p}$-module, where $B_{p}:=B \otimes \mathbb{Q}_{p}$. A polarized abelian $O_{B}$-variety $\underline{A}$ over $k$ is said to be related to $\left(V_{p}, \psi_{p}\right)$ if there is a $B_{p} \otimes L$-linear isomorphism $\alpha: M(\underline{A}) \otimes_{W} L \simeq\left(V_{p}, \psi_{p}\right) \otimes L$ 
which preserves the pairings for a suitable identification $L(1) \simeq L$, where $M(\underline{A})$ is the covariant Dieudonné module with additional structures associated to $\underline{A}$.

Let $G^{\prime}:=\operatorname{GAut}_{B_{p}}\left(V_{p}, \psi_{p}\right)$ be the algebraic group over $\mathbb{Q}_{p}$ of $B_{p}$-linear similitudes. A choice $\alpha$ gives rise to an element $b \in G^{\prime}(L)$ so that one has an isomorphism of isocrystals with additional structures $M(\underline{A}) \otimes L \simeq\left(V_{p} \otimes L, \psi_{p}, b(\mathrm{id} \otimes \sigma)\right)$. The decomposition of $V_{p} \otimes L$ into isoclinic components induces a $\mathbb{Q}$-graded structure, and thus defines a (slope) homomorphism $\nu_{[b]}: \mathbf{D} \rightarrow G^{\prime}$ over some finite extension $\mathbb{Q}_{p^{s}}$ of $\mathbb{Q}_{p}$, where $\mathbf{D}$ is the pro-torus over $\mathbb{Q}_{p}$ with character group $\mathbb{Q}$.

(2) A polarized abelian $O_{B}$-variety $\underline{A}$ is called basic with respect to $\left(V_{p}, \psi_{p}\right)$ if

(a) $\underline{A}$ is related to $\left(V_{p}, \psi_{p}\right)$, and

(b) the slope homomorphism $\nu$ is central.

(3) $\underline{A}$ is called basic if it is basic with respect to $\left(V_{p}, \psi_{p}\right)$ for some skew-Hermitian space $\left(V_{p}, \psi_{p}\right)$.

Lemma 4.4. Let $\underline{A}$ be a polarized abelian $O_{B}$-variety over $k$. The following statements are equivalent.

(a) $\underline{A}$ is basic.

(b) Let $Z$ be the center of $B$ and $Z_{p}=Z \otimes \mathbb{Q}_{p}=\prod_{\mathbf{p} \mid p} Z_{\mathbf{p}}$ be the decomposition as a product of local fields. Let $N=M(\underline{A}) \otimes_{W} L$ be the isocrystals with additional structures associated to $\underline{A}$ and $N=\oplus_{\mathbf{p} \mid p} N_{\mathbf{p}}$ be the decomposition with respect to the $Z_{p}$-action. Then each component $N_{\mathbf{p}}$ is isoclinic.

Proof. See a proof in 6.25 of [14].

One can use the statement (b) of Lemma 4.4 to check whether an object $\underline{A}$ is basic. Note that the statement (b) only depends on the underlying structure of $B$-action, not on the equipped polarization structure. This is also a property of those of arithmetic type; see Lemma 3.6. Indeed, we have

Proposition 4.5. Let $(B, *)$ as in 2.1, An abelian $O_{B}$-variety $\underline{A}=(A, \iota)$ over $k$ is of arithmetic type if and only if it is basic.

Proof. Write $A \sim \prod_{j} A_{i}^{n_{i}}$ into the decomposition up to isogeny with respect to the decomposition $B=\oplus M_{n_{i}}\left(D_{i}\right)$ as in (3.2). By (b) of Lemma $4.4 A$ is basic if and only if each $A_{i}$ is basic. Therefore, we may assume that $B$ is a division algebra.

If $(B, *)$ is of first kind, then by Lemma $4.4 \underline{A}$ is basic if and only if $\underline{A}$ is supersingular. Then this follows from Lemma 4.1 .

Suppose that $(B, *)$ is of second kind. By Lemma 6.28 of Rapoport-Zink [14, $\underline{A}$ is basic if and only there is a finite field $k_{0}$ such that the relative Frobenius morphism $\pi_{A / k_{0}}$ lies in the center $K$ of $B$. The latter statement is equivalent to that the Galois representation $\rho_{\ell}$ factors through the center $Z\left(\operatorname{GAut}_{B}\left(V_{\ell},\langle\rangle,\right)\right)$; see the proof in Theorem 3.12, This completes the proof.

By Proposition 4.5 and Theorem 3.12, we get the following result. This is the motivation of this work.

Theorem 4.6. Let $(B, *)$ as in 2.1. Let $\underline{A}$ be a basic polarized abelian $O_{B}$-variety over an algebraically closed field $k$ of characteristic $p$, and let $N$ be any prime-to-p positive integer. Then

(1) There is a natural bijection

$$
\Lambda_{x, N}(k) \simeq G_{x}(\mathbb{Q}) \backslash G_{x}\left(\mathbb{A}_{f}\right) / U_{N} .
$$


(2) $\operatorname{mass}\left[\Lambda_{x, N}(k)\right]=\operatorname{mass}\left(G_{x}, U_{N}\right)$.

Consider the reduction $\mathcal{M} \otimes \overline{\mathbb{F}}_{p}$ modulo $p$ of a moduli space associated to a PEL-datum $(B, *, V, \psi)$. Let $x=(A, \lambda, \iota, \bar{\eta})$ be a basic point in the moduli space $\mathcal{M} \otimes \overline{\mathbb{F}}_{p}$. By definition, any object $\underline{A}$ in $\mathcal{M}(k)$ satisfies the condition $\left(I_{\ell}\right)$ of (i) for all $\ell \neq p$. The set $\Lambda_{x, N}(k)$ essentially consists of objects in $\mathcal{M}(k)$ satisfying the condition $\left(I_{p}\right)$. The condition $(Q)$ is a mild technical condition which is subject to the Hasse principle; see Remark below for details.

Remark 4.7. (1) We come back to explain the meaning of the conditions (i), (ii) for the set $\Lambda_{x}(k)$ in Subsection 2.1 when $k=\overline{\mathbb{F}}_{p}$. Let $x=\underline{A}_{0}=\left(A_{0}, \lambda_{0}, \iota_{0}\right)$ be a point fixed as before. Then the space consisting of objects $\underline{A}$ that satisfy the condition (i) is the leaf $\mathcal{C}(x)$ passing through $x$ in a moduli space $\mathcal{M} \otimes \overline{\mathbb{F}}_{p}$ of PEL-type. See 12 for the definition of leaves and detail discussions. The moduli space $\mathcal{M} \otimes \overline{\mathbb{F}}_{p}$ is the reduction modulo $p$ of a finite disjoint union of Shimura varieties of PEL-type due to the effect of the Hasse principle. When $x$ is basic, the dimension of the leaf $\mathcal{C}(x)$ is zero. This is also, in fact, a necessary condition for $\operatorname{dim} \mathcal{C}=0$.

(2) The condition (ii) is closely related to the prime-to- $p$ Hecke orbit of $x$. By definition, the prime-to- $p$ Hecke orbit $\mathcal{H}^{(p)}(x)$ consists of objects $\underline{A}$ that satisfy the following conditions

(i) $\operatorname{Isom}_{k}\left(\underline{A}_{0}(\ell), \underline{A}(\ell)\right) \neq \emptyset$ for all $\ell \neq p$, and

(ii) $^{\prime}$ there is a prime-to- $p O_{B}$-linear quasi-isogeny $\varphi: A_{0} \rightarrow A$ which preserves the polarizations.

By definition we have inclusions $\mathcal{H}^{(p)}(x) \subset \Lambda_{x}(k) \subset \mathcal{C}(x)$. When $x$ is basic, or more generally $\underline{A}_{0}$ satisfies the condition

$$
\operatorname{End}_{O_{B}}\left(A_{0}\right) \otimes \mathbb{Z}_{p} \simeq \operatorname{End}_{O_{B}}\left(A_{0}\left[p^{\infty}\right]\right),
$$

then $\mathcal{H}^{(p)}(x)=\Lambda_{x}(k)$. This follows from the weak approximation of the reductive algebraic group $G_{x}$ (defined in Subsection 2.1). Abelian varieties with additional structures that satisfy the condition (4.1) is called B-hypersymmetric in the sense of Chai. Hypersymmetric abelian varieties are studied in Chai and Oort 1 .

(3) When $x$ is basic, $\Lambda_{x}(k)$ in general is strictly smaller than $\mathcal{C}(x)$. That is, the condition (ii) is not automatically satisfied when the condition (i) is satisfied for an object $\underline{A}$. However, it is proved in Rapoport and Zink [14, Chapter 6] that if $\underline{A}$ satisfies the condition (i), then there is an associated class $[\underline{A}]$ in $H^{1}\left(\mathbb{Q}, G_{x}\right)$ such that this class vanishes if and only if the condition (ii) is also satisfied. Moreover, since $\underline{A}$ satisfies the condition (i), the class $[\underline{A}]$ lies in the kernel $\operatorname{ker}^{1}\left(\mathbb{Q}, G_{x}\right)$ of the local-global map

$$
H^{1}\left(\mathbb{Q}, G_{x}\right) \rightarrow \prod_{v} H^{1}\left(\mathbb{Q}_{v}, G_{x}\right)
$$

where $v$ runs through all places of $\mathbb{Q}$. Indeed, the map $\underline{A} \mapsto[\underline{A}]$ gives a bijection between the set of quasi-isogeny classes of objects $\underline{A}$ satisfying the condition (i) and the $\operatorname{set}^{\operatorname{ker}^{1}}\left(\mathbb{Q}, G_{x}\right)$. In particular, when $G_{x}$ satisfies the Hasse principle, one has the quality $\Lambda_{x}(k)=\mathcal{C}(x)$. This is the case for the type $\mathrm{C}$ family of Shimura varieties because $G_{x}$ is an inner form of the defining reductive group $G_{1}$, which is semi-simple and simply connected. The reader may find that in [16] and [17] the condition (ii) is not imposed; this is because the Hasse principle for $G_{x}$ is satisfied. In general, we do need to impose such a condition, which replaces the failure of the Hasse principle. 


\section{REFERENCES}

[1] C.-L. Chai and F. Oort, Hypersymmetric abelian varieties. Pure Appl. Math. Q. 2 (2006), $1-27$.

[2] A.J. de Jong, Homomorphisms of Barsotti-Tate groups and crystals in positive characteristic. Invent. Math. 134 (1998), 301-333.

[3] T. Ekedahl, On supersingular curves and supersingular abelian varieties. Math. Scand. 60 (1987), 151-178.

[4] G. Faltings, Endlichkeitssätze für abelsche Varietäten über Zahlkörpern. Invent. Math. 73 (1983), 349-366.

[5] G. Faltings, G. Wüstholz, F. Grunewald, N. Schappacher and U. Stuhler, Rational points. With an appendix by Wüstholz. Aspects of Mathematics, E6. Friedr. Vieweg ES Sohn, Braunschweig, 1992.

[6] K. Hashimoto and T. Ibukiyama, On class numbers of positive definite binary quaternion hermitian forms, J. Fac. Sci. Univ. Tokyo 27 (1980), 549-601.

[7] T. Ibukiyama, T. Katsura and F. Oort, Supersingular curves of genus two and class numbers. Compositio Math. 57 (1986), 127-152.

[8] R. E. Kottwitz, Isocrystals with additional structure. Compositio Math. 56 (1985), 201-220.

[9] R. E. Kottwitz, Points on some Shimura varieties over finite fields. J. Amer. Math. Soc. 5 (1992), 373-444.

[10] D. Mumford, Abelian Varieties. Oxford University Press, 1974.

[11] A. Ogus, Supersingular K3 crystals. Journées de Géométrie. Algébrique de Rennes (1978), Vol II, pp. 3-86, Astérisque, no. 64. Soc. Math. France, Paris, 1979.

[12] F. Oort, Foliations in moduli spaces of abelian varieties. J. Amer. Math. Soc. 17 (2004), $267-296$.

[13] F. Oort, Minimal p-divisible groups. Ann. Math. 161 (2005), 1021-1036.

[14] M. Rapoport and Th. Zink, Period Spaces for p-divisible groups. Ann. Math. Studies 141, Princeton Univ. Press, 1996.

[15] J.-P. Serre, Résumé des cours de 1984-1985. pp. 27-32, CEuvres Collected papers. IV. 19851998, 2000.

[16] C.-F. Yu, On the supersingular locus in Hilbert-Blumenthal 4-folds. J. Algebraic Geom. 12 (2003), 653-698.

[17] C.-F. Yu, On the mass formula of supersingular abelian varieties with real multiplications. $J$. Australian Math. Soc. 78 (2005), 373-392.

[18] J.G. Zarhin, Isogenies of abelian varieties over fields of finite characteristics, Math. USSR Sbornik 24 (1974), 451-461.

Institute of Mathematics, Academia Sinica, 128 Academia Rd. Sec. 2, Nankang, Taipei, TAiwan And NCTS (TAIPEi OfFice)

E-mail address: chiafu@math.sinica.edu.tw

Max-Planck-Institut für Mathematik, Vivatsgasse 7, Bonn, 53111, Germany 